\title{
Frame Dragging, vorticity and electromagnetic fields in axially symmetric stationary spacetimes
}

\author{
L Herrera ${ }^{1} \dagger$, G A González ${ }^{2} \ddagger$, L A Pachón ${ }^{2,3} \S$ and J A Rueda ${ }^{2} \|$ \\ ${ }^{1}$ Escuela de Física, Universidad Central de Venezuela, Caracas, Venezuela. \\ ${ }^{2}$ Escuela de Física, Universidad Industrial de Santander, A.A. 678, Bucaramanga, \\ Colombia. \\ ${ }^{3}$ Laboratorio de Astronoma y Física Teórica (LAFT), Departamento de Física, \\ Facultad de Ciencias, La Universidad del Zulia, Maracaibo, 4004, Venezuela
}

\begin{abstract}
We present a general study about the relation between the vorticity tensor and the Poynting vector of the electromagnetic field for axially symmetric stationary electrovacuum metrics. The obtained expressions allow to understand the role of the Poynting vector in the dragging of inertial frames. The particular case of the rotating massive charged magnetic dipole is analyzed in detail. In addition, the electric and magnetic parts of the Weyl tensor are calculated and the link between the later and the vorticity is established. Then we show that, in the vacuum case, the necessary and sufficient condition for the vanishing of the magnetic part is that the spacetime be static.
\end{abstract}

\section{Introduction}

The dragging of inertial frames produced by self-gravitating sources is a general relativistic effect and as such is of utmost relevance in the search of observational support of the theory. Therefore it is important to study its occurrence under a variety of circumstances. Some years ago, Bonnor [1] studied the behaviour of a time-like congruence in the spacetime produced by a static massive charged magnetic dipole using an approximate solution of the Einstein-Maxwell equations.

$\dagger$ e-mail: laherrera@telcel.net.ve

$\ddagger$ e-mail: guillego@uis.edu.co

$\S$ e-mail: lpachon@laft.org

|| e-mail: jrueda@tux.uis.edu.co 
Surprisingly, a frame dragging effect appears in such spacetime. To explain such an effect, Bonnor invokes the existence of a non-vanishing electromagnetic Poynting vector in the equatorial plane of the source.

As we shall show in this work, that suggestion is correct and, indeed, it is the Poynting vector the responsible for the appearance of vorticity in the timelike congruence. It should be stressed that in [1] the electromagnetic potentials are $\phi=e X^{-1}$ and $\vec{A}=-\mu \rho^{2} X^{-3 / 2} \hat{e}_{\phi}$, where $X=\sqrt{\rho^{2}+z^{2}}$, which do not generate proportional electric and magnetic fields. Now, according to Das [2], the electric and magnetic fields of a static solution must be proportional. Therefore the solution considered in [1] is stationary but not static.

In this paper we shall present a general study of the frame dragging in the stationary axisymmetric case by analyzing the vorticity tensor and its eventual relationship with the Poynting vector associated with the electromagnetic field generated by the source. For this purpose, we shall consider the exact solution of the Einstein-Maxwell equations describing the rotating massive charged magnetic dipole. This solution is analyzed in some limiting cases, and we shall focus on the case when the angular momentum of the source is zero, i.e., the solution describing the massive charged magnetic dipole.

We shall also calculate the magnetic and electric parts of the Weyl tensor for the axially symmetric stationary case. The study of the electric $E_{\alpha \beta}$ and magnetic $H_{\alpha \beta}$ parts of Weyl tensor has attracted the attention of researchers for many years (see [3] -23] and references therein). Particularly intriguing is the eventual relationship of

the magnetic part of the Weyl tensor with rotation [4, 12, 6] and with gravitational radiation [3, 8, 11, 15, 16, 17. In order to delve deeper into these issues, we shall analyze the role of the electric and the magnetic part of the Weyl tensor in the vorticity in the special case of vacuum stationary axisymmetric spacetimes. From the obtained expressions, it follows that the vanishing of the magnetic part implies that the spacetime is static, i.e., we show that the vorticity tensor vanishes if and only if the magnetic part of the Weyl tensor vanishes.

\section{Vorticity Tensor in Axistationary Einstein-Maxwell Spacetimes}

The simplest line element representing a stationary axisymmetric spacetime is given by $[24,25]$

$$
d s^{2}=-f(d t-\omega d \phi)^{2}+f^{-1}\left[e^{2 \gamma}\left(d \rho^{2}+d z^{2}\right)+\rho^{2} d \phi^{2}\right]
$$


where the metric functions $f, \omega$ and $\gamma$ are functions of the Weyl coordinates $\rho$ and $z$. The vorticity tensor for a congruence with tangent vector $u^{\alpha}$ is defined as

$$
\omega_{\alpha \beta}=u_{[\alpha ; \beta]}+\dot{u}_{[\alpha} u_{\beta]},
$$

where $\dot{u}_{\alpha}$ is the acceleration and $\omega_{\alpha \beta}$ is the rotation or vorticity of the congruence [25]. For a congruence of observers at rest in the frame of (1), the four-velocity is defined by the timelike eigenvector

$$
u^{\alpha}=\left(\frac{1}{\sqrt{f}}, 0,0,0\right),
$$

and the vorticity tensor is given by

$$
\omega_{\alpha \beta}=\left(\begin{array}{cccc}
0 & 0 & 0 & 0 \\
0 & 0 & 0 & -\frac{1}{2} \sqrt{f} \omega_{, \rho} \\
0 & 0 & 0 & -\frac{1}{2} \sqrt{f} \omega_{, z} \\
0 & \frac{1}{2} \sqrt{f} \omega_{, \rho} & \frac{1}{2} \sqrt{f} \omega_{, z} & 0
\end{array}\right) .
$$

In order to establish the physical meaning of the relationship between the vorticity tensor and the metric function $\omega$, we shall analyze the problem with the aid of the Ernst potentials $\mathcal{E}$ and $\Phi$ [26]

$$
\begin{aligned}
& \mathcal{E}(\rho, z)=f-\Phi \Phi^{*}+i \Omega, \\
& \Phi(\rho, z)=\phi+i \psi,
\end{aligned}
$$

where $\psi$ and $\Omega$ are defined through the relations

$$
\begin{aligned}
& \rho^{-1} f(\nabla \phi-\omega \nabla A)=\hat{e}_{\phi} \times \nabla \psi, \\
& \nabla \cdot\left\{f^{-2}\left[\nabla \Omega+2 \operatorname{Im}\left(\Phi^{*} \nabla \Phi\right)\right]\right\}=0,
\end{aligned}
$$

respectively. Here $\phi$ and $A$ are, respectively, the time and azimuthal components of the electromagnetic four-potential $A_{\alpha}$.

Using the Ernst potentials $\mathcal{E}$ and $\Phi$ the Einstein-Maxwell equations read

$$
\begin{aligned}
f & =\operatorname{Re} \mathcal{E}+\Phi \Phi^{*}, \\
\omega_{, \rho} & =-\rho f^{-2} \operatorname{Im}\left(\mathcal{E}_{, z}+2 \Phi^{*} \Phi_{, z}\right), \\
\omega_{, z} & =\rho f^{-2} \operatorname{Im}\left(\mathcal{E}_{, \rho}+2 \Phi^{*} \Phi_{, \rho}\right), \\
\gamma_{, \rho} & =\frac{\rho}{4\left(\operatorname{Re} \mathcal{E}+\Phi \Phi^{*}\right)^{2}}\left[\left(\mathcal{E}_{, \rho}+2 \Phi^{*} \Phi_{, \rho}\right)\left(\mathcal{E}_{, \rho}^{*}+2 \Phi \Phi_{, \rho}^{*}\right)\right. \\
& \left.-\left(\mathcal{E}_{, z}+2 \Phi^{*} \Phi_{, z}\right)\left(\mathcal{E}_{, z}^{*}+2 \Phi \Phi_{, z}^{*}\right)\right]-\frac{\rho\left(\Phi_{, \rho} \Phi_{, \rho}^{*}-\Phi_{, z} \Phi_{, z}^{*}\right)}{\operatorname{Re} \mathcal{E}+\Phi \Phi^{*}},
\end{aligned}
$$




$$
\gamma_{, z}=\frac{\rho \operatorname{Re}\left[\left(\mathcal{E}_{, \rho}+2 \Phi \Phi_{, \rho}^{*}\right)\left(\mathcal{E}_{, z}^{*}+2 \Phi \Phi_{, z}^{*}\right)\right]}{2\left(\operatorname{Re} \mathcal{E}+\Phi \Phi^{*}\right)^{2}}-\frac{2 \rho \operatorname{Re}\left(\Phi_{, \rho}^{*} \Phi_{, z}\right)}{\operatorname{Re} \mathcal{E}+\Phi \Phi^{*}} .
$$

Now, by using the expressions for $\omega$ given by (10) in (40) the vorticity tensor can be written as

$$
\omega_{\alpha \beta}=\omega_{\alpha \beta}^{\Omega}+\omega_{\alpha \beta}^{\Phi},
$$

where

$$
\begin{aligned}
& \omega_{\alpha \beta}^{\Omega}=\frac{1}{2} \rho f^{-3 / 2}\left(\begin{array}{cccc}
0 & 0 & 0 & 0 \\
0 & 0 & 0 & \Omega_{, z} \\
0 & 0 & 0 & -\Omega_{, \rho} \\
0 & -\Omega_{, z} & \Omega_{, \rho} & 0
\end{array}\right), \\
& \omega_{\alpha \beta}^{\Phi}=\rho f^{-3 / 2}\left(\begin{array}{cccc}
0 & 0 & 0 & 0 \\
0 & 0 & 0 & \operatorname{Im}\left(\Phi^{*} \Phi_{, z}\right) \\
0 & 0 & 0 & -\operatorname{Im}\left(\Phi^{*} \Phi_{, \rho}\right) \\
0 & -\operatorname{Im}\left(\Phi^{*} \Phi_{, z}\right) & \operatorname{Im}\left(\Phi^{*} \Phi_{, \rho}\right) & 0
\end{array}\right) .
\end{aligned}
$$

\section{Physical Meaning of the tensors $\omega_{\alpha \beta}^{\Omega}$ and $\omega_{\alpha \beta}^{\Phi}$}

The theory of relativistic multipole moments [27, 28, 29] for stationary axisymmetric spacetimes was developed using $\xi$ and $q$ potentials

$$
\mathcal{E}=\frac{1-\xi}{1+\xi}, \quad \Phi=\frac{q}{1+\xi},
$$

which are the analogues of Newtonian gravitational potential and Coulomb potential respectively. The real part of the $\xi$ potential describes the matter distribution and its imaginary part describes the source rotations. On the other hand, the real part of the $q$ potential is related with the electric field and its imaginary part with the magnetic field.

The multipole moments over the whole space-time are given by the coefficients of the series expansion of $\tilde{\xi}$ and $\tilde{q}$ as power of $\bar{\rho}, \bar{z}$ :

$$
\tilde{\xi}=\sum_{i, j=0}^{\infty} a_{i j} \bar{\rho}^{i} \bar{z}^{j}, \quad \tilde{q}=\sum_{i, j=0}^{\infty} b_{i j} \bar{\rho}^{i} \bar{z}^{j},
$$

where $\tilde{\xi}$ and $\tilde{q}$ denote the $\xi$ potential and the $q$ potential in the conformal space given by the coordinate transformation

$$
\bar{\rho}=\frac{\rho}{\rho^{2}+z^{2}}, \quad \bar{z}=\frac{z}{\rho^{2}+z^{2}}, \quad \bar{\phi}=\phi .
$$


Thus, writing the $\xi$ potential as

$$
\xi=M+i J
$$

the rotational multipole moments are given by the coefficients of the series expansion of $J$. Combining the expressions (5), (15) and (18) we obtain

$$
J=-\frac{2 \Omega}{|1+\mathcal{E}|^{2}} .
$$

From the above expression we can identify the $\Omega$ potential as the responsible for the rotational multipole moments; i.e., if the $\Omega$ potential vanishes all rotational multipole moments also vanish. In general relativity the gravitational field is affected by the electromagnetic field and by the mass rotations (angular momentum). The $\Omega$ potential is also affected by these factors and therefore it contains contributions from both (angular momentum and electromagnetic field). This explains why the $\Omega$ potential does not necessarily vanish in the case when the angular momentum of the source is zero but electromagnetic fields are present.

Therefore, the $\omega_{\alpha \beta}^{\Omega}$ tensor has a clear physical meaning, it gives the contribution of the source rotations to the vorticity of the congruence.

Next, using the definition of the $\Phi$ potential given by (6), the $\omega_{\alpha \beta}^{\Phi}$ tensor is

$$
\omega_{\alpha \beta}^{\Phi}=\rho f^{-3 / 2}\left(\begin{array}{cccc}
0 & 0 & 0 & 0 \\
0 & 0 & 0 & \phi \psi_{, z}-\psi \phi_{, z} \\
0 & 0 & 0 & -\left(\phi \psi_{, \rho}-\psi \phi_{, \rho}\right) \\
0 & -\left(\phi \psi_{, z}-\psi \phi_{, z}\right) & \phi \psi_{, \rho}-\psi \phi_{, \rho} & 0
\end{array}\right) .
$$

This tensor vanishes when either one of the potentials vanishes or in the general case when the potentials $\phi$ and $\psi$ are proportional to each other. So, the $\omega_{\alpha \beta}^{\Phi}$ tensor exists only in the case when the electric and magnetic fields coexist (being not proportional to each other) and therefore we can conclude that this tensor gives the contribution of the electromagnetic field to the vorticity of the congruence. To clarify the physical meaning of the tensors $\omega_{\alpha \beta}^{\Omega}$ and $\omega_{\alpha \beta}^{\Phi}$ we shall now study the vorticity of the exact solution of the Einstein-Maxwell for a rotating massive charged dipolar magnetic source.

\section{The spacetime around a rotating massive charged magnetic dipole}

The solution of the Einstein-Maxwell equations that describes the spacetime around a rotating massive charged magnetic dipole was given by Manko in [30]. The Ernst 
potentials on the symmetry axis, $\mathcal{E}(\rho=0, z)=e(z)$ and $\Phi(\rho=0, z)=f(z)$, were chosen as

$$
e(z)=\frac{z-m-i a}{z+m-i a}, \quad f(z)=\frac{q z+i b}{z(z+m-i a)} .
$$

The physical meaning of the parameters $m, a, q$ and $b$ is derived from the Simon multipole moments [29]. The first moments are calculated for (21) with the aid of the Hoenselaers-Perjés procedure [27] and have the form

$$
\begin{aligned}
& P_{0}=m, \quad P_{1}=i a m, \quad P_{2}=-a^{2} m \quad P_{3}=-i a^{3} m, \\
& P_{4}=m a^{4}+\frac{1}{70}\left(3 b^{2}+13 a b q\right) m, \quad P_{5}=i a^{5} m-i \frac{1}{21}\left(b^{2}-6 a b q\right) a m \\
& Q_{0}=q, \quad Q_{1}=i(b+a q), \quad Q_{2}=-a(b+a q), \quad Q_{3}=-i a^{2}(b+a q), \\
& Q_{4}=a^{3}(b+a q)+\frac{1}{70}\left(10 b q(b+a q)+3 a b m^{2}\right), \\
& Q_{5}=i a^{4}(b+a q)-\frac{1}{21} i(b+a q)\left(b^{2}-6 a b q\right) .
\end{aligned}
$$

To obtain a representation of the solution in terms of determinants we shall rewrite the axis data (21) in the form

$$
e(z)=1+\frac{e_{1}}{z-\beta_{1}}, \quad f(z)=\frac{f_{1}}{z-\beta_{1}}+\frac{f_{2}}{z},
$$

where

$$
e_{1}=-2 m, \quad \beta_{1}=-m+i a, \quad f_{1}=\frac{m q-i(b+a q)}{m-i a}, \quad f_{2}=\frac{i b}{m-i a} .
$$

The Ernst potentials over the whole spacetime can be obtained using the general formulae describing the extended multisoliton electrovac solution [31] and are written as

$$
\mathcal{E}=\frac{E_{+}}{E_{-}}, \quad \Phi=\frac{F}{E_{-}}
$$

with

$$
E_{ \pm}=\left|\begin{array}{rcccc}
1 & 1 & 1 & 1 & 1 \\
\pm 1 & \frac{r_{1}}{\alpha_{1}-\beta_{1}} & \frac{r_{2}}{\alpha_{2}-\beta_{1}} & \frac{r_{3}}{\alpha_{3}-\beta_{1}} & \frac{r_{4}}{\alpha_{4}-\beta_{1}} \\
\pm 1 & \frac{r_{1}}{\alpha_{1}} & \frac{r_{2}}{\alpha_{2}} & \frac{r_{3}}{\alpha_{3}} & \frac{r_{4}}{\alpha_{4}} \\
0 & \frac{h_{1}\left(\alpha_{1}\right)}{\alpha_{1}-\beta_{1}^{*}} & \frac{h_{1}\left(\alpha_{2}\right)}{\alpha_{2}-\beta_{1}^{*}} & \frac{h_{1}\left(\alpha_{3}\right)}{\alpha_{3}-\beta_{1}^{*}} & \frac{h_{1}\left(\alpha_{4}\right)}{\alpha_{4}-\beta_{1}^{*}} \\
0 & \frac{h_{2}\left(\alpha_{1}\right)}{\alpha_{1}-\beta_{1}^{*}} & \frac{h_{2}\left(\alpha_{2}\right)}{\alpha_{2}-\beta_{1}^{*}} & \frac{h_{2}\left(\alpha_{3}\right)}{\alpha_{3}-\beta_{1}^{*}} & \frac{h_{2}\left(\alpha_{4}\right)}{\alpha_{4}-\beta_{1}^{*}}
\end{array}\right|
$$




$$
F=\left|\begin{array}{rcccc}
1 & f\left(\alpha_{1}\right) & f\left(\alpha_{2}\right) & f\left(\alpha_{3}\right) & f\left(\alpha_{4}\right) \\
-1 & \frac{r_{1}}{\alpha_{1}-\beta_{1}} & \frac{r_{2}}{\alpha_{2}-\beta_{1}} & \frac{r_{3}}{\alpha_{3}-\beta_{1}} & \frac{r_{4}}{\alpha_{4}-\beta_{1}} \\
-1 & \frac{r_{1}}{\alpha_{1}} & \frac{r_{2}}{\alpha_{2}} & \frac{r_{3}}{\alpha_{3}} & \frac{r_{4}}{\alpha_{4}} \\
0 & \frac{h_{1}\left(\alpha_{1}\right)}{\alpha_{1}-\beta_{1}^{*}} & \frac{h_{1}\left(\alpha_{2}\right)}{\alpha_{2}-\beta_{1}^{*}} & \frac{h_{1}\left(\alpha_{3}\right)}{\alpha_{3}-\beta_{1}^{*}} & \frac{h_{1}\left(\alpha_{4}\right)}{\alpha_{4}-\beta_{1}^{*}} \\
0 & \frac{h_{2}\left(\alpha_{1}\right)}{\alpha_{1}-\beta_{1}^{*}} & \frac{h_{2}\left(\alpha_{2}\right)}{\alpha_{2}-\beta_{1}^{*}} & \frac{h_{2}\left(\alpha_{3}\right)}{\alpha_{3}-\beta_{1}^{*}} & \frac{h_{2}\left(\alpha_{4}\right)}{\alpha_{4}-\beta_{1}^{*}}
\end{array}\right| .
$$

The $\alpha_{i}$ parameters are the roots of the Sibgatullin's equation, i.e. the roots of

$$
e(z)+e^{*}(z)+2 f(z) f^{*}(z)=0,
$$

with * denoting complex conjugation. The other parameters are defined as

$$
\begin{aligned}
& r_{k}=\sqrt{\rho^{2}+\left(z-\alpha_{k}\right)^{2}}, \quad f\left(\alpha_{j}\right)=\frac{f_{1}}{\alpha_{j}-\beta_{1}}+\frac{f_{2}}{\alpha_{j}}, \\
& h_{1}\left(\alpha_{j}\right)=e_{1}^{*}+2 f_{1}^{*} f\left(\alpha_{1}\right), \quad h_{2}\left(\alpha_{j}\right)=2 f_{1}^{*} f\left(\alpha_{1}\right),
\end{aligned}
$$

The metric functions $f, \omega$, and $\gamma$ and the Kinnersley potential $\mathcal{K}$ are given by

$$
\begin{aligned}
& f=\frac{E_{+} E_{-}^{*}+E_{-} E_{+}^{*}+2 F F^{*}}{2 E_{-} E_{-}^{*}}, \quad e^{2 \gamma}=\frac{E_{+} E_{-}^{*}+E_{-} E_{+}^{*}+2 F F^{*}}{2 K_{0} K_{0}^{*} r_{1} r_{2} r_{3} r_{4}}, \\
& \omega=\frac{2 \operatorname{Im}\left(E_{-} H^{*}-E_{-}^{*} G-F I^{*}\right)}{E_{+} E_{-}^{*}+E_{-} E_{+}^{*}+2 F F^{*}}, \quad \mathcal{K}=-i \frac{I}{E_{-}}
\end{aligned}
$$

with

$$
\begin{aligned}
& G=\left|\begin{array}{rcccc}
1 & g_{1} & g_{2} & g_{3} & g_{4} \\
-1 & \frac{r_{1}}{\alpha_{1}-\beta_{1}} & \frac{r_{2}}{\alpha_{2}-\beta_{1}} & \frac{r_{3}}{\alpha_{3}-\beta_{1}} & \frac{r_{4}}{\alpha_{4}-\beta_{1}} \\
-1 & \frac{r_{1}}{\alpha_{1}} & \frac{r_{2}}{\alpha_{2}} & \frac{r_{3}}{\alpha_{3}} & \frac{r_{4}}{\alpha_{4}} \\
0 & \frac{h_{1}\left(\alpha_{1}\right)}{\alpha_{1}-\beta_{1}^{*}} & \frac{h_{1}\left(\alpha_{2}\right)}{\alpha_{2}-\beta_{1}^{*}} & \frac{h_{1}\left(\alpha_{3}\right)}{\alpha_{3}-\beta_{1}^{*}} & \frac{h_{1}\left(\alpha_{4}\right)}{\alpha_{4}-\beta_{1}^{*}} \\
0 & \frac{h_{2}\left(\alpha_{1}\right)}{\alpha_{1}-\beta_{1}^{*}} & \frac{h_{2}\left(\alpha_{2}\right)}{\alpha_{2}-\beta_{1}^{*}} & \frac{h_{2}\left(\alpha_{3}\right)}{\alpha_{3}-\beta_{1}^{*}} & \frac{h_{2}\left(\alpha_{4}\right)}{\alpha_{4}-\beta_{1}^{*}}
\end{array}\right| \\
& I=\left|\begin{array}{cccccc}
f_{1}+f_{2} & 0 & f\left(\alpha_{1}\right) & f\left(\alpha_{2}\right) & f\left(\alpha_{3}\right) & f\left(\alpha_{4}\right) \\
z & 1 & 1 & 1 & 1 & 1 \\
-\beta_{1} & -1 & \frac{r_{1}}{\alpha_{1}-\beta_{1}} & \frac{r_{2}}{\alpha_{2}-\beta_{1}} & \frac{r_{3}}{\alpha_{3}-\beta_{1}} & \frac{r_{4}}{\alpha_{4}-\beta_{1}} \\
0 & -1 & \frac{r_{1}}{\alpha_{1}} & \frac{r_{2}}{\alpha_{2}} & \frac{r_{3}}{\alpha_{3}} & \frac{r_{4}}{\alpha_{4}} \\
e_{1}^{*} & 0 & \frac{h_{1}\left(\alpha_{1}\right)}{\alpha_{1}-\beta_{1}^{*}} & \frac{h_{1}\left(\alpha_{2}\right)}{\alpha_{2}-\beta_{1}^{*}} & \frac{h_{1}\left(\alpha_{3}\right)}{\alpha_{3}-\beta_{1}^{*}} & \frac{h_{1}\left(\alpha_{4}\right)}{\alpha_{4}-\beta_{1}^{*}} \\
0 & 0 & \frac{h_{2}\left(\alpha_{1}\right)}{\alpha_{1}-\beta_{1}^{*}} & \frac{h_{2}\left(\alpha_{2}\right)}{\alpha_{2}-\beta_{1}^{*}} & \frac{h_{2}\left(\alpha_{3}\right)}{\alpha_{3}-\beta_{1}^{*}} & \frac{h_{2}\left(\alpha_{4}\right)}{\alpha_{4}-\beta_{1}^{*}}
\end{array}\right| \\
& H=\left|\begin{array}{ccccc}
z & 1 & 1 & 1 & 1 \\
\beta_{1} & \frac{r_{1}}{\alpha_{1}-\beta_{1}} & \frac{r_{2}}{\alpha_{2}-\beta_{1}} & \frac{r_{3}}{\alpha_{3}-\beta_{1}} & \frac{r_{4}}{\alpha_{4}-\beta_{1}} \\
0 & \frac{r_{1}}{\alpha_{1}} & \frac{r_{2}}{\alpha_{2}} & \frac{\frac{r_{3}}{\alpha_{3}}}{\alpha_{4}} & \frac{r_{4}}{\alpha_{4}} \\
e_{1}^{*} & \frac{h_{1}\left(\alpha_{1}\right)}{\alpha_{1}-\beta_{1}^{*}} & \frac{h_{1}\left(\alpha_{2}\right)}{\alpha_{2}-\beta_{1}^{*}} & \frac{h_{1}\left(\alpha_{3}\right)}{\alpha_{3}-\beta_{1}^{*}} & \frac{h_{1}\left(\alpha_{4}\right)}{\alpha_{4}-\beta_{1}^{*}} \\
0 & \frac{h_{2}\left(\alpha_{1}\right)}{\alpha_{1}-\beta_{1}^{*}} & \frac{h_{2}\left(\alpha_{2}\right)}{\alpha_{2}-\beta_{1}^{*}} & \frac{h_{2}\left(\alpha_{3}\right)}{\alpha_{3}-\beta_{1}^{*}} & \frac{h_{2}\left(\alpha_{4}\right)}{\alpha_{4}-\beta_{1}^{*}}
\end{array}\right|, \quad K_{0}=\left|\begin{array}{cccc}
\frac{1}{\alpha_{1}-\beta_{1}} & \frac{1}{\alpha_{2}-\beta_{1}} & \frac{1}{\alpha_{3}-\beta_{1}} & \frac{1}{\alpha_{4}-\beta_{1}} \\
\frac{1}{\alpha_{1}} & \frac{1}{\alpha_{2}} & \frac{1}{\alpha_{3}} & \frac{1}{\alpha_{4}} \\
\frac{h_{1}\left(\alpha_{1}\right)}{\alpha_{1}-\beta_{1}^{*}} & \frac{h_{1}\left(\alpha_{2}\right)}{\alpha_{2}-\beta_{1}^{*}} & \frac{h_{1}\left(\alpha_{3}\right)}{\alpha_{3}-\beta_{1}^{*}} & \frac{h_{1}\left(\alpha_{4}\right)}{\alpha_{4}-\beta_{1}^{*}} \\
\frac{h_{2}\left(\alpha_{1}\right)}{\alpha_{1}-\beta_{1}^{*}} & \frac{h_{2}\left(\alpha_{2}\right)}{\alpha_{2}-\beta_{1}^{*}} & \frac{h_{2}\left(\alpha_{3}\right)}{\alpha_{3}-\beta_{1}^{*}} & \frac{h_{2}\left(\alpha_{4}\right)}{\alpha_{4}-\beta_{1}^{*}}
\end{array}\right| .
\end{aligned}
$$




\section{Relation between the Poynting Vector and the Vorticity tensor}

The electromagnetic Poynting vector is defined as $S^{i}=-T^{i \alpha} u_{\alpha}$, being $T^{\alpha \beta}$ the electromagnetic energy-momentum tensor

$$
T_{\alpha \beta}=\frac{1}{4 \pi}\left(-g^{\gamma \delta} F_{\alpha \gamma} F_{\beta \delta}+\frac{1}{4} g_{\alpha \beta} F_{\gamma \delta} F^{\gamma \delta}\right),
$$

where $F_{\alpha \beta}$ is the electromagnetic field tensor $F_{\alpha \beta}=2 A_{[\beta ; \alpha]}$ and $A_{\mu}=(\phi, 0, A, 0)$ is the electromagnetic four-potential. For this case, the only non-vanishing component of the Poynting vector is

$$
S^{\phi}=\frac{f^{3 / 2}}{\pi \rho^{2} e^{2 \gamma}}(\omega \nabla \phi \cdot \nabla \phi+\nabla \phi \cdot \nabla A),
$$

where the potential $\phi$ is the real part of the Ernt potential $\Phi$ and $A$ is the real part of the Kinnersley potential $\mathcal{K}$

$$
\phi=\operatorname{Re}\left(\frac{F}{E_{-}}\right), \quad A=\operatorname{Re}\left(-i \frac{I}{E_{-}}\right) .
$$

¿From (29) and (26) we obtain the following expressions

$$
\begin{aligned}
S^{\phi}= & \frac{2\left(E_{+} E_{-}^{*}+E_{-} E_{+}^{*}+2 F F^{*}\right)^{1 / 2} K_{0} K_{0}^{*} r_{1} r_{2} r_{3} r_{4}}{\pi \rho^{2}\left(2 E_{-} E_{-}^{*}\right)^{3 / 2}}\left\{2 \operatorname{Im}\left[\frac{E_{-} H^{*}-E_{-}^{*} G-F I^{*}}{E_{+} E_{-}^{*}+E_{-} E_{+}^{*}+2 F F^{*}}\right] \times\right. \\
& {\left[\operatorname{Re}^{2}\left[\frac{E_{-} F_{, \rho}-F\left(E_{-}\right)_{, \rho}}{2 E_{-} E_{-}}\right]+\operatorname{Re}^{2}\left[\frac{E_{-} F_{, z}-F\left(E_{-}\right)_{, z}}{2 E_{-} E_{-}}\right]\right]+\operatorname{Re}\left[\frac{E_{-} F_{, \rho}-F\left(E_{-}\right)_{, \rho}}{2 E_{-} E_{-}}\right] \times } \\
& \left.\operatorname{Re}\left[\frac{E_{-} I_{, \rho}-I\left(E_{-}\right)_{, \rho}}{2 i E_{-} E_{-}}\right]+\operatorname{Re}\left[\frac{E_{-} F_{, z}-F_{\left(E_{-}\right), z}}{2 E_{-} E_{-}}\right] \operatorname{Re}\left[\frac{E_{-} I_{, z}-I\left(E_{-}\right)_{, z}}{2 i E_{-} E_{-}}\right]\right\},
\end{aligned}
$$

for the Poynting vector, and

$$
\begin{aligned}
& \Phi^{*} \Phi_{, \rho}=\left[\frac{E_{-} F_{, \rho}-F\left(E_{-}\right)_{, \rho}}{E_{-} E_{-}}\right] \frac{F^{*}}{E_{-}^{*}}, \quad \Phi^{*} \Phi_{, z}=\left[\frac{E_{-} F_{, z}-F\left(E_{-}\right)_{, z}}{E_{-} E_{-}}\right] \frac{F^{*}}{E_{-}^{*}} \\
& \Omega_{, \rho}=\left[\frac{E_{-}\left(E_{+}\right), \rho-E_{+}\left(E_{-}\right)_{, \rho}}{E_{-} E_{-}}\right], \quad \Omega_{, z}=\left[\frac{E_{-}\left(E_{+}\right)_{, z}-E_{+}\left(E_{-}\right)_{, z}}{E_{-} E_{-}}\right] .
\end{aligned}
$$

for the quantities in the vorticity tensor.

\subsection{Analysis in some limiting cases}

In order to study the relations between the vorticity tensor and the Poynting vector, we shall consider, in this section, some limiting cases of the above presented solution. 
5.1.1. Spinning mass $(q=0, b=0)$. This case represents the Kerr solution for which the vorticity tensor and the Poynting vector are given by

$$
\omega_{\alpha \beta}^{\Omega} \neq 0, \quad \omega_{\alpha \beta}^{\Phi}=0, \quad S^{\phi}=0 .
$$

In this case we have not electromagnetic fields. Therefore it is obvious that the contributions to the vorticity due to the tensor $\omega_{\alpha \beta}^{\Phi}$ and the Poynting vector vanish, and so the frame dragging is related to $\omega_{\alpha \beta}^{\Omega}$.

5.1.2. Massive magnetic dipole $(a=0, q=0)$. This case reduces to the static dipole magnetic solution derived by Gutsunnaev and Manko [32] and we have

$$
\omega_{\alpha \beta}^{\Omega}=0, \quad \omega_{\alpha \beta}^{\Phi}=0, \quad S^{\phi}=0 .
$$

Here we have neither monopole electric field nor electric or magnetic fields generated by higher order multipole moments, because there are no angular moment. Obviously, no frame dragging occurs in this case.

5.1.3. Charged mass $(a=0, b=0)$. This case reduces to the Reissner-Nordström solution and we obtain

$$
\omega_{\alpha \beta}^{\Omega}=0, \quad \omega_{\alpha \beta}^{\Phi}=0, \quad S^{\phi}=0 .
$$

The above results are obvious because the Reissner-Nordström solution is static.

5.1.4. Spinning massive magnetic dipole $(q=0)$. This case reduces to the stationary generalization of the Kerr metric corresponding to a magnetized spinning mass derived by Manko [33. The vorticity tensor and the Poynting vector are characterized by

$$
\omega_{\alpha \beta}^{\Omega} \neq 0, \quad \omega_{\alpha \beta}^{\Phi} \neq 0, \quad S^{\phi} \neq 0 .
$$

Here, even though we have not electric field generated by a monopole of charge, we have an electric field generated by higher order multipole moments induced by the spinning magnetic dipole.

5.1.5. Spinning charged mass $(b=0)$. This case represents the Kerr-Newmann solution, for which we have

$$
\omega_{\alpha \beta}^{\Omega} \neq 0, \quad \omega_{\alpha \beta}^{\Phi} \neq 0, \quad S^{\phi} \neq 0 .
$$

For this case the rotation of the mass and the electrical monopole induce a magnetic field, producing a non-vanishing Poynting vector. 
5.1.6. Massive charged magnetic dipole $(a=0)$. This is the case considered by Bonnor using an approximate solution [1]. Thus we have

$$
\omega_{\alpha \beta}^{\Omega} \neq 0, \quad \omega_{\alpha \beta}^{\Phi} \neq 0, \quad S^{\phi} \neq 0 .
$$

The parameter $a$ in (21) is responsible, on the symmetry axis, for the multipole moments of rotation (22) and its absence produces the vanishing of $\Omega$ potential on the symmetry axis, but not over the whole spacetime. The electromagnetic field contributes to this potential making it non vanishing over there, for this case we have

$$
\left.\Omega\right|_{a=0}=\operatorname{Im} \frac{A-B}{A+B},
$$

where

$$
\begin{aligned}
A & =b\left[m^{2}-q^{2}\right]\left[\kappa_{+}^{2}\left(R_{+} r_{-}+R_{-} r_{+}\right)+\kappa_{-}^{2}\left(R_{+} r_{+}+R_{-} r_{-}\right)\right]-4 b^{3}\left(R_{+} R_{-}\right. \\
& \left.+r_{+} r_{-}\right)+i b q\left[\kappa_{+}\left(R_{+} r_{-}-R_{-} r_{+}\right)-\kappa_{-}\left(R_{+} r_{+}-R_{-} r_{-}\right)\right] \kappa_{+} \kappa_{-} \\
B & =m \kappa_{+} \kappa_{-}\left\{b \left[\kappa_{+} \kappa_{-}\left(R_{+}+R_{-}+r_{+}+r_{-}\right)-\left(m^{2}-q^{2}\right)\left(R_{+}+R_{-}-r_{+}\right.\right.\right. \\
& \left.\left.\left.-r_{-}\right)\right]-i b q\left[\left(\kappa_{+}+\kappa_{-}\right)\left(r_{+}-r_{-}\right)+\left(\kappa_{+}-\kappa_{-}\right)\left(R_{-}-R_{+}\right)\right]\right\}
\end{aligned}
$$

with

$R_{ \pm}=\sqrt{\rho^{2}+\left[z \pm\left(\kappa_{+}+\kappa_{-}\right)\right]^{2}}, \quad r_{ \pm}=\sqrt{\rho^{2}+\left[z \pm\left(\kappa_{+}-\kappa_{-}\right)\right]^{2}}, \quad \kappa_{ \pm}=\sqrt{m^{2}-q^{2} \pm 2 b}$.

¿From the above we can see that when the angular moment is zero, the stationary character of the field is given by a term that is proportional to $b q$. This term could be interpreted as the rotation of the electric charge $q$ in presence of a magnetic dipole $b$ and it is the responsible for the vorticity in absence of mass rotations.

From the limiting cases it is clear that the only possible configurations have the following features:

- $\omega_{\alpha \beta}^{\Omega}=\omega_{\alpha \beta}^{\Phi}=0$. This configuration corresponds to the static case. It is represented by solutions of non-rotating sources of vacuum and by the electromagnetostatic case, in which the electric and magnetic field are proportional [2].

- $\omega_{\alpha \beta}^{\Omega} \neq 0, \omega_{\alpha \beta}^{\Phi}=0$. This configuration corresponds to the stationary vacuum case.

- $\omega_{\alpha \beta}^{\Omega} \neq 0, \omega_{\alpha \beta}^{\Phi} \neq 0$. This configuration describes the stationary electrovacuum case.

So far, we have focused on the study of the vorticity tensor. However, the magnetic part of the Weyl tensor has been usually related with gravitational radiation and with the vorticity of the congruence of the observers. Therefore, in the next section, we shall study the possible relation between these tensors in the stationary axisymmetric vacuum case. 


\section{The electric and magnetic parts of Weyl tensor}

The electric and magnetic parts of Weyl tensor, $E_{\alpha \beta}$ and $H_{\alpha \beta}$, respectively, are formed from the Weyl tensor $C_{\alpha \beta \gamma \delta}$ and its dual $\tilde{C}_{\alpha \beta \gamma \delta}$ by contraction with the timelike vector given above:

$$
\begin{aligned}
& E_{\alpha \beta}=C_{\alpha \gamma \beta \delta} u^{\gamma} u^{\delta}, \\
& H_{\alpha \beta}=\tilde{C}_{\alpha \gamma \beta \delta} u^{\gamma} u^{\delta} .
\end{aligned}
$$

Then, if for some global smooth unit timelike vector field such as $u^{a}$ the Weyl tensor of a given spacetime $(\mathcal{M}, g)$ satisfies

$$
E_{\alpha \beta}=0,
$$

we say that this is a Purely Magnetic Weyl Spacetime (PMWS). On the other hand, if the Weyl tensor satisfies

$$
H_{\alpha \beta}=0 .
$$

we say that this is a Purely Electric Weyl Spacetime (PEWS).

For the Papapetrou spacetime (II) the non vanishing components of the electric Weyl tensor $E_{\alpha \beta}$ are

$$
\begin{aligned}
E_{11} & =\frac{1}{6 \rho^{2} f}\left[3 \rho^{2}\left(f_{, z} \gamma_{, z}-f_{, \rho} \gamma_{, \rho}\right)-\rho^{2} f\left(\gamma_{, \rho \rho}+\gamma_{, z z}\right)+\rho^{2}\left(2 f_{, \rho \rho}-f_{, z z}\right)\right. \\
& \left.+f^{3}\left(\omega_{, \rho}^{2}-2 \omega_{, z}^{2}\right)+3 \rho f \gamma_{, \rho}-\rho f_{, \rho}\right], \\
E_{12} & =E_{21}=-\frac{1}{\rho^{2} f}\left[\rho^{2}\left(f_{, \rho} \gamma_{, z}-f_{, z} \gamma_{, \rho}\right)-\rho^{2} f_{, z \rho}-f^{3} \omega_{, z} \omega_{, \rho}-\rho f \gamma_{, z}\right], \\
E_{22} & =\frac{1}{6 \rho^{2} f}\left[3 \rho^{2}\left(f_{, z} \gamma_{, z}-f_{, \rho} \gamma_{, \rho}\right)+\rho^{2} f\left(\gamma_{, \rho \rho}+\gamma_{, z z}\right)+\rho^{2}\left(f_{, \rho \rho}-2 f_{, z z}\right)\right. \\
& \left.+f^{3}\left(2 \omega_{, \rho}^{2}-\omega_{, z}^{2}\right)+3 \rho f \gamma_{, \rho}+\rho f_{, \rho}\right], \\
E_{33} & =\frac{1}{6 f e^{2 \gamma}}\left[f^{3}\left(\omega_{, \rho}^{2}+\omega_{, z}^{2}\right)+2 \rho^{2} f\left(\gamma_{, \rho \rho}+\gamma_{, z z}\right)-\rho^{2}\left(f_{, \rho \rho}+f_{, z z}\right)+2 \rho f_{, \rho}\right],
\end{aligned}
$$

whereas

$$
\begin{aligned}
H_{11} & =\frac{1}{2 \rho^{2}}\left[\omega_{, \rho}\left(\rho f_{, z}-\rho f \gamma_{, z}\right)+\omega_{, z}\left(2 \rho f_{, \rho}-\rho f \gamma_{, \rho}-f\right)+f \rho \omega_{, z \rho}\right], \\
H_{12} & =H_{21}=\frac{1}{4 \rho^{2}}\left[\omega_{, \rho}\left(f+2 \rho f \gamma_{, \rho}-3 \rho f_{, \rho}\right)+\omega_{, z}\left(3 \rho f_{, z}-2 \rho f \gamma_{, z}\right)\right. \\
& \left.+\rho f\left(\omega_{, z z}-\omega_{, \rho \rho}\right)\right], \\
H_{22} & =-\frac{1}{2 \rho}\left[\omega_{, \rho}\left(2 f_{, z}-f \gamma_{, z}\right)+\omega_{, z}\left(f_{, \rho}-f \gamma_{, \rho}\right)+f \omega_{, \rho z}\right], \\
H_{33} & =\frac{1}{e^{2 \gamma}}\left[\rho f_{, z} \omega_{, \rho}+\omega_{, z}\left(f-\rho f_{, \rho}\right)\right],
\end{aligned}
$$

are the non vanishing components of the magnetic Weyl tensor $H_{\alpha \beta}$. 


\section{Purely electric Weyl vacuum solutions. Vanishing conditions for $H_{\alpha \beta}$}

In this section we will show the following theorem:

Theorem 1. If $(\mathcal{M}, g)$ is an axisymmetric vacuum spacetime endowed with a timelike Killing vector, then the magnetic part of the Weyl tensor vanishes along the direction defined by the stationary Killing vector, if and only if $(\mathcal{M}, g)$ is a static axisymmetric spacetime.

Now, a stationary spacetime is static if its temporal Killing vector $\partial_{t}$ is hypersurface orthogonal. For the line element (11), this property is achieved if the metric function $\omega$ is zero. Therefore, the theorem can be stated as

$$
H_{\alpha \beta}=0 \Leftrightarrow \omega=0 .
$$

We shall first show (i) $\omega=0 \Rightarrow H_{\alpha \beta}=0$, and after that (ii) $H_{\alpha \beta}=0 \Rightarrow \omega=0$.

(i) If $\omega$ is constant, it is easy to see that all the components of the magnetic part of Weyl tensor vanish, because every component depends on the derivatives of the $\omega$ function. On the other hand, in order to satisfy regularity conditions on the symmetry axis, this constant value of the metric function $\omega$ must be zero. Therefore, we have that

$$
\omega=0 \Rightarrow H_{\alpha \beta}=0 \text {. }
$$

(ii) To show that $H_{\alpha \beta}=0 \Rightarrow \omega=0$, we use the Einstein vacuum field written as

$$
\begin{aligned}
& f \nabla^{2} f=\nabla f \cdot \nabla f-\rho^{-2} f^{4} \nabla \omega \nabla \omega, \\
& \nabla \cdot\left(\rho^{-2} f^{2} \nabla \omega\right)=0, \\
& 4 \gamma_{, \rho}=\rho f^{-2}\left(f_{, \rho}^{2}-f_{, z}^{2}\right)-\rho^{-1} f^{2}\left(\omega_{, \rho}^{2}-\omega_{, z}^{2}\right), \\
& 2 \gamma_{, z}=\rho f^{-2} f_{, \rho} f_{, z}-\rho^{-1} f^{2} \omega_{, \rho} \omega_{, z} .
\end{aligned}
$$

Then, from $H_{33}=0$, we have that

$$
\rho^{2}\left(\rho^{-1} f\right)_{, z}-\rho^{2}\left(\rho^{-1} f\right)_{, \rho} \omega_{, z}=0 .
$$

This equation can be written in the form

$$
\left[\nabla\left(\rho^{-1} f\right) \times \nabla \omega\right] \cdot \hat{e}_{\phi}=0
$$

whose general solution is

$$
\nabla(\tilde{f}) \times \nabla \omega=0, \quad \tilde{f}=\rho^{-1} f .
$$

Equation (60) implies, at least, one of the following conditions:
(a) $\nabla \omega=0$,
(b) $\nabla \tilde{f}=0$
(c) $\omega$ is of the form $\omega=F(\tilde{f})$. 
Case (a): If the gradient of $\omega$ is equal to zero then $\omega$ is a constant which, as was mentioned before, must be $\omega=0$.

Case (b): The Einstein field equations (54)-(57) in terms of $\tilde{f}$ read

$$
\begin{aligned}
& \tilde{f} \nabla^{2} \tilde{f}=\nabla \tilde{f} \cdot \nabla \tilde{f}-\tilde{f}^{4} \nabla \omega \cdot \nabla \omega, \\
& \nabla \cdot\left(\tilde{f}^{2} \nabla \omega\right)=0, \\
& 4 \gamma_{, \rho}=\rho^{-1}+\rho \tilde{f}^{-2} \tilde{f}_{, \rho}^{2}+2 \tilde{f}^{-1} \tilde{f}_{, \rho}^{2}-\rho \tilde{f}^{-2} \tilde{f}_{, z}^{2}-\rho \tilde{f}^{2}\left(\omega_{, \rho}^{2}-\omega_{, z}^{2}\right), \\
& 2 \gamma_{, z}=\tilde{f}^{-1} \tilde{f}_{, z}+\rho \tilde{f}^{-2} \tilde{f}_{, \rho} \tilde{f}_{, z}-\rho \tilde{f}^{2} \omega_{, \rho} \omega_{, z} .
\end{aligned}
$$

Using the fact that in this case $\tilde{f}$ is constant, it follows at once from (61) that

$$
\nabla \omega=0 .
$$

which brings us back to the case (a) above.

Case (c): In this case, using the notation $F^{\prime} \equiv \frac{d F}{d \tilde{f}}$, the gradient and the Laplacian of the $\omega$ function are

$$
\nabla \omega=F^{\prime} \nabla \tilde{f}, \quad \nabla^{2} \omega=F^{\prime} \nabla^{2} \tilde{f}+F^{\prime \prime} \nabla \tilde{f} \cdot \nabla \tilde{f} .
$$

Replacing the above result into (62), we obtain

$$
\tilde{f} F^{\prime} \nabla^{2} \tilde{f}=-\left(\tilde{f} F^{\prime \prime}+2 F^{\prime}\right) \nabla \tilde{f} \cdot \nabla \tilde{f} .
$$

Next, using (66) and (67) in (61) we obtain

$$
\left[\tilde{f} F^{\prime \prime}-\tilde{f}^{4}\left(F^{\prime}\right)^{3}+3 F^{\prime}\right] \nabla \tilde{f} \cdot \nabla \tilde{f}=0,
$$

implying, either

(1) $\nabla \tilde{f}=0$

or,

(2) $\tilde{f} F^{\prime \prime}-\tilde{f}^{4}\left(F^{\prime}\right)^{3}+3 F^{\prime}=0$.

The case (1) brings us back to (b), producing $\omega$ equal to zero.

For the case (2) we have to solve the corresponding non-linear differential equation. Making the substitution $u=\tilde{f}^{3} F^{\prime}$, the solution of that equation reads

$$
\frac{1}{u^{2}}=\frac{1}{\tilde{f}^{2}}+K_{1}
$$

where $K_{1}$ is an integration constant which has to be choosen equal to zero, since in the limit $u \rightarrow \infty$, we have $\tilde{f} \rightarrow \infty$ too, because $F^{\prime} \neq 0$, so the solution is

$$
F=\omega=\mp \frac{1}{\tilde{f}}+K_{2}
$$


where $K_{2}$ is another integration constant. Without lost of generality we may assume $K_{2}=0$. Therefore, the $\omega$ function is

$$
\omega=\frac{1}{\tilde{f}}=\frac{\rho}{f} .
$$

Now, replacing (171) in (61) we obtain

$$
\tilde{f} \nabla^{2} \tilde{f}=0, \quad \nabla^{2} \tilde{f}=0(\tilde{f} \text { cannot be zero }) .
$$

Next, replacing (171) in (56) and (57) we obtain

$$
\begin{aligned}
& 4 \gamma_{, \rho}=-\rho^{-1}+2 f^{-1} f_{, \rho}, \\
& 2 \gamma_{, z}=f^{-1} f_{, z},
\end{aligned}
$$

and replacing (71), (73) and (74) in the components of magnetic part of the Weyl tensor (48) which are assumed to vanish, it follows

$$
\begin{aligned}
& f_{, z \rho}=\frac{f_{, z}}{4 \rho}, \\
& 2\left(f_{, z z}-f_{, \rho \rho}\right)+\left(\rho^{-1} f\right)_{, \rho}=0,
\end{aligned}
$$

or, in terms of $\tilde{f}$,

$$
\begin{aligned}
& \left(4 \rho \tilde{f}_{, \rho}+3 \tilde{f}\right)_{, z}=0, \\
& 2\left(\tilde{f}_{, z z}-\rho^{-1} \tilde{f}_{, \rho}-\tilde{f}_{, \rho \rho}\right)-\rho^{-1} \tilde{f}_{, \rho}=0 .
\end{aligned}
$$

Thus, the the vanishing of the magnetic part of the Weyl tensor $H_{\alpha \beta}$ implies (77) and (178).

In addition, the function $\tilde{f}$ must be a solution of the Laplace equation (72)

$$
\tilde{f}_{\rho \rho}+\rho^{-1} \tilde{f}_{, \rho}+\tilde{f}_{, z z}=0 .
$$

Using the above equation in (78) we obtain the following differential equation

$$
4 \tilde{f}_{, \rho \rho}+5 \rho^{-1} \tilde{f}_{, \rho}=0
$$

whose solution is

$$
\tilde{f}(\rho, z)=-\frac{4 h_{1}(z)}{\rho^{1 / 4}}+h_{2}(z),
$$

where $h_{1}$ and $h_{2}$ are functions of the coordinate $z$ only.

Replacing (81) in (77), it follows

$$
8 \frac{d h_{1}}{d z}-3 \rho^{1 / 4} \frac{d h_{2}}{d z}=0,
$$

whose only, obvious, solution is

$$
h_{1}^{\prime}=h_{2}^{\prime}=0 \Leftrightarrow h_{1}=a, \quad h_{2}=b, \quad a, b \text { are constants . }
$$


with $h^{\prime} \equiv \frac{d h}{d z}$. So the function $\tilde{f}$ becomes

$$
\tilde{f}=-4 a \rho^{-1 / 4}+b .
$$

The constants $a$ and $b$ can be calculated by replacing $\tilde{f}$ in the Laplace equation. Doing so we obtain $a=0$, implying

$$
\tilde{f}=b=\text { constant },
$$

This result brings us back to the case (b). Therefore, it has been established that

$$
H_{\alpha \beta}=0 \Rightarrow \omega=0 \text {. }
$$

This last result, together with the conclusion from the point (i), prove the theorem enunciated before.

It is worth noticing that, since the necessary and sufficient condition for the simultaneous vanishing of the magnetic part of the Weyl tensor and the vorticity tensor in the vacuum case is that the metric function $\omega$ vanishes, we may write from the above

$$
H_{\alpha \beta}=0 \Leftrightarrow \omega_{\alpha \beta}=0 .
$$

In the general electrovacuum case we were unable to establish a relationship like this. However, it can be esaily inferred from (4) and (48) that when the space-time is static $(\omega=0)$ the vorticity tensor and the magnetic part of the Weyl tensor also vanish.

\section{Conclusions}

We have seen so far, that the vorticity tensor may be splitted in two parts: one of them is directly related to rotational multipole moments, whereas the other represents the contribution from the electromagnetic field.

In all the analyzed limiting cases for the rotating massive charged magnetic dipole, it appears that the electromagnetic contribution to the vorticity vanishes whenever the corresponding Poynting vector does. This fact support the Bonnor's idea, that it is the electromagnetic energy flux on the equatorial plane, the responsible for the dragging effect observed in the field of a massive charged magnetic dipole.

In the vacuum case it was established that the necessary and sufficient condition for a stationary axisymmetric spacetime to be static is the vanishing of the magnetic part of Weyl tensor. This complements and generalizes previous results indicating that vacuum static spacetimes are purely electric [9, 34. This result can also be expressed through a direct relationship between the magnetic part of the Weyl tensor and the vorticity tensor.

\section{Acknowledgments}

G.A.G. wants to thank the financial support from COLCIENCIAS, Colombia. 


\section{References}

[1] Bonnor W. B. 1991 Phys. Lett. A 15823

[2] Das A 1979 J. Math. Phys. 120740

[3] Bel L 1962 Cah. de Phys. 1659 ; 2000 Gen. Rel. Grav. 322047

[4] Glass E N 1975 J. Math. Phys. 162361

[5] Barnes A and Rowlingson R 1989 Class. Quantum Grav. 6949

[6] De Felice F and Clarke C J S 1990 Relativity on curved manifolds (Cambridge University Press) pp 254-5

[7] Matarrese S , Pantano O and Saez D 1993 Phys. Rev. D 471311

[8] Bruni M , Matarrese S and Pantano O 1995 Astrophys. J 445958

[9] McIntosh C, Arianrhod R, Wade S and Hoenselaers C 1994 Class. Quantum Grav. 111555

[10] Haddow B M 1995 J. Math. Phys. 181378

[11] Bonnor W B 1995 Class. Quantum. Grav. 12499

[12] Bonnor W B 1995 Class. Quantum. Grav. 121483

[13] van Elst H, C Uggla, Lesame W M , Ellis G F R and Maartens R 1997 Class. Quantum Grav $14,1151$.

[14] van Elst H and Ellis G F R 1998 Class. Quantum Grav. 15, 3545.

[15] Dunsby P K S, Basset B A and Ellis G F R 1997 Class. Quantum. Grav. 141215

[16] Maartens R, Ellis G F R and Siklos T 1997 Class. Quantum. Grav. 141927

[17] Hogan P and Ellis G F R 1997 Class. Quantum. Grav. 14 A171

[18] Maartens R and Basset B 1998 Class. Quantum Grav. 15705.

[19] Maartens R, Lesame W M and Ellis G F R 1998 Class. Quantum. Grav. 151005

[20] Lozanowski C and Aarons M 1999 Class. Quantum. Grav. 164075

[21] N Van den Bergh 2003 Class. Quantum. Grav. 20 L1; 2003 Class. Quantum. Grav. 20 L165

[22] Ferrando J and Saez J 2003 Class. Quantum. Grav. 202835

[23] Herrera L, Santos N. O. and Carot J. 2005 "Gravitational radiation, vorticity and the electric and magnetic part of Weyl tensor" $g r-q c / 0511112$

[24] Papapetrou A 1953 Ann. Phys. 12, 309

[25] Stephani H, Kramer D, MacCallum M, Honselaers C and Herlt E 2003 Exact Solutions to Einstein's Field Equations. Second Edition, (Cambridge University Press, Cambridge),

[26] Ernst F J 1968 Phys. Rev. 1681415

[27] Hoenselaers C and Perjés Z 1990 Class. Quantum Grav. 71819

[28] Fodor G, Hoenselaers C and Perjés Z 1989 J. Math. Phys. 302252

[29] Simon W 1984 J. Math. Phys. 251035

[30] Manko V S 1993 Phys. Lett.181 349

[31] Manko V S, Martin J. and E. Ruíz 1995 Phys. Rev. D 514187

[32] Gutsunnaev T I and Manko V S 1987 Phys. Lett. A 123215

[33] Manko V S 1993 Class. Quantum Grav.10 L239

[34] Ehlers J and Kundt W 1962 Gravitation: An Introduction to Current Research ed. L. Witten (New York: Wiley) 